\title{
Wormhole Detection Algorithm: To Detect Wormhole Attacks Efficiently in Wireless Networks
}

\author{
R. Regan ${ }^{1}$, E. Dhivyadarsani ${ }^{2}$, B. Nandhini ${ }^{3}$ and A. Priya ${ }^{4}$ \\ University College of Engineering Villupuram, India \\ ${ }^{1}$ reganr85@gmail.com,2dhivyaelango30@gmail.com, \\ ${ }^{3}$ nandhininandhu533@gmail.com, ${ }^{4}$ lashmipriya@gmail.com
}

\begin{abstract}
The way of remote specially appointed and sensor systems make them exceptionally alluring to aggressors. A standout amongst the most prevalent and genuine assaults in remote impromptu systems is wormhole assault and most proposed conventions to protect against this assault utilized situating gadgets, synchronized timekeepers, or directional reception apparatuses. We propose a novel calculation for identifying worm-opening assaults in remote multi-bounce systems. The proposed methodology is totally limited and, not at all like numerous procedures proposed in writing, does not utilize any exceptional equipment antique or area data, making the strategy all around appropriate. The calculation is autonomous of remote correspondence models. Be that as it may, learning of the model and hub dispersion gauges a parameter utilized as a part of the calculation. We display reproduction results for three diverse correspondence models and two distinctive hub circulations, and demonstrate that the calculation can recognize wormhole assaults with $100 \%$ discovery and $0 \%$ false caution probabilities at whatever point the system is associated with high likelihood. Notwithstanding for low thickness systems where a possibility of disengagement is high, the recognition likelihood stays high.
\end{abstract}

Keywords: RTT, DWA, Wormhole attack

\section{Introduction}

ADHOC and sensor systems are developing as a promising stage for an assortment of use ranges in both military and regular citizen spaces. Notwithstanding, the open way of the remote correspondence channels, the absence of foundation, the quick organization rehearses, and the unfriendly situations where they might be sent, make them powerless against an extensive variety of security assaults. Among these assaults wormhole assault is difficult to identify on the grounds that this assault does not infuse unusual volumes of movement into the system. In this work, a particular sort of rising security risk knows as the wormhole assault is explored. Remote specially appointed and sensor systems are commonly utilized out as a part of an open, uncontrolled environment, frequently in antagonistic domains. Specifically, a few critical applications for such systems originate from military and resistance enclosures. Utilization of remote medium and innate synergistic nature of the system conventions make such system defenseless against different types of assaults. In this paper our emphasis is on an especially destroying type of assault, called wormhole assault [1]-[3]. Here, the foe associates two far off focuses in the system utilizing an immediate low-inactivity join called the wormhole join. The wormhole connection can be set up by an assortment of means, e.g., by utilizing a system link and any type of "wired" connection innovation or a long-go remote transmission in an alternate band. The end-purposes of this connection (wormhole hubs) are furnished with radio handsets perfect with the specially appointed or sensor system to be assaulted. 
Once the wormhole connection is set up, the foe catches remote transmissions toward one side, sends them through the wormhole interface and replays them at the flip side.

\section{Related Work}

In [2] creators have considered bundle rope - geographic and fleeting. In geographic rope, hub area data is utilized to bound the separation a parcel can navigate. Since wormhole assaults can influence limitation, the area data must be gotten by means of an out-of-band component, for example, GPS. Further, the "legitimate" separation a bundle can navigate is not generally simple to decide. In transient chains, to a great degree precise all around synchronized tickers are utilized to bound the proliferation time of bundles that could be difficult to acquire $\mathrm{n}$ especially in ease sensor equipment. Notwithstanding when accessible, such timing examination will most likely be unable to recognize slice through or physical layer wormhole assaults. In [1], a confirmed separation bouncing system called MAD is utilized. The methodology is like parcel rope at an abnormal state, yet does not require area data or clock synchronization. Be that as it may, despite everything it experiences different restrictions of the bundle rope procedure. In the Echo convention [4], ultrasound is utilized to head the separation for secure area confirmation. Utilization of ultrasound rather than RF signals as before aides in unwinding the timing prerequisites; however needs an extra equipment. In a late work [4], creators have concentrated on down to earth strategies for identifying wormholes. This method utilizes timing imperatives and verification to check whether a hub is a genuine neighbor. The creators build up a convention that can be actualized in 802.11 fit equipment with minor changes. Still it stays hazy how reasonable such timing investigation could be in minimal effort sensor equipment.

\section{Limitations of Prior Work and Our Contributions}

The present answers for wormhole are restricted especially regarding extensive sensor systems, where sensor hubs convey ease, moderately unsophisticated equipment and scale-capacity is an imperative configuration objective. These principles out utilization of extra equipment antiquity that few reported methods use -, for example, directional reception apparatuses [7], GPS [2], ultrasound [8], protect hubs with right area [5]. This additionally precludes fine grain timing investigation utilized as a part of a few procedures [2], [4]. Additionally, physical-layer assaults might be safe to timing investigation [4]. At last, the adaptability prerequisites preclude worldwide clock synchronization [2] or any type of worldwide calculations [1]. In the present work, we build up a limited calculation for distinguishing wormhole assaults that is absolutely in light of neighborhood network data. Such data is regularly gathered any path by different upper layer conventions, for example, directing, in this manner may not introduce any extra overhead. No extra equipment antique is required making the methodology generally pertinent. No timing examination is done guaranteeing that we can identify even physical layer assaults. Our system does not utilize area data and can recognize assaults that are propelled even before the system is set up, that may impact restriction. We expect that our procedure is especially helpful for sensor systems as the current methods are very constrained there. Additionally, availability is not anticipated that would change oftentimes in sensor systems, making our network based approach entirely down to earth. The discovery calculation basically searches for prohibited substructures in the availability charts that ought not be available in a lawful network diagram. Comprehension of the remote correspondence model (i.e., a model that depicts with some given certainty whether a connection between two hubs ought to exist) helps the identification calculation significantly, however is not entirely required. 


\section{Wormhole Attack}

In the wormhole assault [6,], a vindictive hub burrows messages got in one a player in the system over a low idleness interface and replays them in an alternate part. Because of the way of remote transmission, the assailant can make a wormhole notwithstanding for bundles not tended to itself, since it can catch them in remote transmission and passage them to the conspiring aggressor at the inverse end of the wormhole. The passage can be built up in a wide range of routes, for example, through an out-of band shrouded channel (e.g., a wired connection), parcel exemplification, or powerful transmission. The passage makes the figment that the two end focuses are near each other, by making burrowed parcels arrive either sooner or with lesser number of jumps contrasted with the bundles sent over ordinary courses. This permits an assailant to subvert the right operation of the directing convention, by controlling various courses in the system. Later, he can utilize this to perform activity investigation or specifically drop information movement. The wormhole assault mostly comprises in system layer assaults when the assault is arranged by convention stacks. Examined the making of the wormhole and stances three ways:

1) Tunneling the bundles over the system layer

2) Long Range burrow utilizing high power transmitters

3) Tunnel creation through wired foundation

\section{System Architecture}

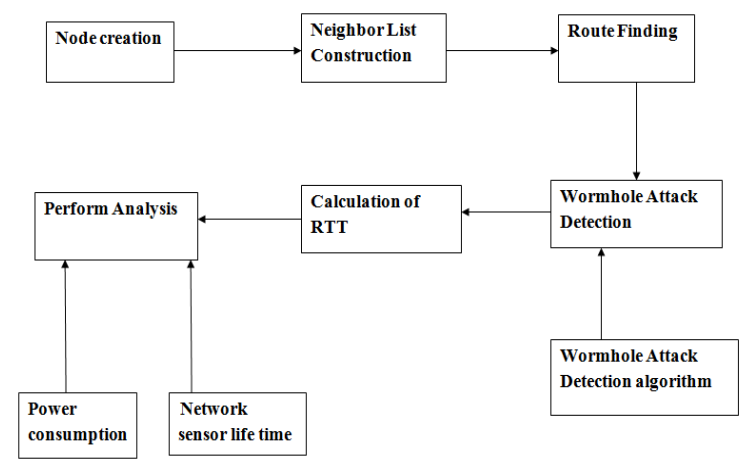

Figure 1. System Architecture

\section{Wormhole Detection Algorithm}

The position of wormhole impacts the system network by making long connections between two arrangements of hubs found possibly far away. The subsequent availability diagram along these lines goes astray from the genuine network chart. Our discovery calculation basically searches for taboo substructures in the availability chart that ought not be available in a lawful network diagram. Learning of the remote correspondence model between the hubs helps our location calculation. This is on the grounds that a correspondence model can characterize what substructures saw in the availability chart could be taboo. How-ever, our methodology is still material when the correspondence model is obscure. For this situation we have to run an additional pursuit methodology to decide a basic parameter for the location calculation. This parameter will be clarified later in this segment. We first build up our wormhole recognition calculation, beginning from the unit plate chart model and after that general (known or obscure) correspondence 
models, lastly talks about how to naturally uproot joins made by wormhole once a wormhole is identified.

Modules

- Neighbor List Construction

- Route Finding

- Wormhole Attack Detection

- Calculation of RTT

- Perform analysis

\section{A. Neighbour List Construction}

In this first stage, every hub show the neighbor demand (NREQ) message. The NREQ accepting hub reacts to the neighbor answer (NREP) message. The asking for hub develops the neighbor records in view of the got of NREP messages and tallies its neighbor number (nn). After that the source hub begins the course development stage.

\section{B. Route Finding}

At that stage, the source hub is dependable to develop the various leveled directing tree to different hubs in the sensor field. The hub sends the course ask for (RREQ) message to the neighbor hub and recovery the season of its RREQ sending TREQ. The halfway hub additionally advances the RREQ message and saves TREQ of its sending time. At the point when the RREQ message achieves the destination hub, it sends course answer message (RREP) with the saved way. The RTT can be ascertained as:

$$
\mathrm{RTT}=T_{R E P}-T_{R E Q}
$$

\section{Wormhole Attack Detection}

In this stage, the source hub figures the RTT of every single transitional hub furthermore it and destination. It figures the RTT of progressive hubs and looks at the worth to check whether the wormhole assault can be there or not. In the event that there is no assault, the estimations of them are almost the same. On the off chance that the RTT quality is higher than other progressive hubs, it can be suspected as wormhole assault between this connection. The following recognition system depends on the way that by bringing new connections into the system chart, the enemy builds the quantity of neighbors of the hubs inside of its sweep. So it needs to check the $n$ of these two hubs which appraise normal number of neighbors $d$. It is approximated as $d=(\mathrm{N}-1) \pi \mathrm{r} 2 / \mathrm{A}$ where an is the territory of the area, $\mathrm{N}$ is the quantity of hubs in that locale and $\mathrm{r}$ is the basic transmission range. For instance, if the RTT esteem between A to B is impressively more noteworthy than for different connections, it needs to check the estimation of $\mathrm{nn}$ for An and B. On the off chance that likewise the nn esteem for An and B is higher than the normal neighbor number $\mathrm{d}$, there is a suspect that a wormhole connection is between hubs An and B. Along these lines the component can pinpoint the area of the wormhole assault.

\section{Calculation of RTT}

In this subsection, the point by point estimation of the RTT is talked about. The estimation of RTT is viewed as the time contrast between a hub gets RREP from a destination to it send RREQ to the destination. Amid course setup system, the season of sending RREQ and getting RREP is portrayed. For this situation, each hub will spare the 
time they forward RREQ and the time they get RREP from the destination to ascertain the RTT. Given all RTT values between hubs in the course and the destination, RTT between two progressive hubs, say An and B, can be figured as takes after: RTTA,B= RTTARTTB Where RTTA is the RTT between hub An and the destination, RTTB is the RTT between hub $\mathrm{B}$ and the destination. For instance, the course from source (S) to destination (D) go through hub An, and B so which steering way incorporates: $\mathrm{S} \rightarrow \mathrm{A} \rightarrow \mathrm{B} \rightarrow \mathrm{D}$

\section{E. Performance Analysis}

In this area, the execution of the proposed component is assessed utilizing system test system (ns2). In this test, the system incorporates 50 hubs conveyed haphazardly in a 1000 meters $\times 1000$ meters field and the transmission reach is characterized 250 meters. There is no development of hubs and the foundation movement is created haphazardly by an arbitrary generator gave by ns2. The CBR association with 4 parcels for each second are made and the extent of the bundle is 512 bytes. In the reproduction, two wormhole hubs are made haphazardly into the system and set up a passage between them utilizing exemplification.

\section{Algorithm Description}

Review that the wormhole discovery calculation is to seek by every hub a prohibited structure in its neighborhood. The calculation is limited and circulated. Every hub looks for prohibited structures in its k-jump neighborhood. We will clarify the calculation for the general $k$-jump location. In our exact studies $k \leq 2$ was discovered adequate for a large portion of the cases. Every hub u keeps up the rundown of $2 \mathrm{k}$-bounce neighbors N2k (u). Hub u finds a non-neighboring hub, $\mathrm{v}$, fromN2k (u) and checks their k-bounce neighbor records to figure their normal k-jump neighbors $\mathrm{Ck}(\mathrm{u}, \mathrm{v})$. Note that to discover a nonunfilled $\mathrm{Ck}(\mathrm{u}, \mathrm{v})$ set, hub u need not search for v beyond2khops. We now need to search for the presence of the taboo substructure (i.e., fk free hubs) in $\mathrm{Ck}(\mathrm{u}, \mathrm{v})$. One approach to do this would be to register the most extreme autonomous set among $\mathrm{Ck}(\mathrm{u}, \mathrm{v})$ and contrasting the measure of this set and fk. In any case, processing the most extreme free set is a NP-difficult issue, notwithstanding for unit circle diagrams [18], [19]. In this manner we unwind the recognition standard by finding a maximal autonomous set (an arrangement of free hubs such that no other hub can be incorporated), which should be possible by a straightforward avaricious calculation: we begin from an unfilled set, pick a self-assertive hub and incorporate it in the free set, evacuate its neighbors, and proceed until we come up short on hubs in $\mathrm{Ck}(\mathrm{u}, \mathrm{v})$. The subsequent set is a maximal autonomous set. We think about the extent of the maximal autonomous set along these lines got with the prohibited parameter $\mathrm{k}$. In the event that it is equivalent or bigger than $\mathrm{f} \mathrm{k}$, then we yield 'wormhole identified'. The framework of the calculation is as per the following.

1) In a preprocessing stage, locate the prohibited parameter fk, in view of the hub appropriation and correspondence model. (For UDGs, the bound on fk can be gotten from Lemmas 3.1 and 3.2. We examine different procedures of finding fk by and by in the following subsection, which likewise sum up to non-UDGs.)

2) Each hub u decides its $2 \mathrm{k}$-jump neighbor rundown, N2k (u), and executes the accompanying strides for each non-neighboring hub $\mathrm{v}$ in $\mathrm{N} 2 \mathrm{k}(\mathrm{u})$.

3) Node $u$ decides the arrangement of regular k-jump neighbors with $v$ from their kbounce neighbor records. This is $\mathrm{Ck}(\mathrm{u}, \mathrm{v})=\mathrm{Nk}(\mathrm{u}) \cap \mathrm{Nk}(\mathrm{v})$. This can be controlled by basically trading neighbor records.

4) Node $u$ decides the maximal autonomous arrangement of the sub-diagram on vertices $\mathrm{Ck}(\mathrm{u}, \mathrm{v})$, by utilizing the eager calculation displayed previously. 
5) If the maximal free set size is equivalent or bigger than $\mathrm{fk}$, hub $\mathrm{u}$ declares the nearness of a wormhole.

The way the calculation is introduced makes it show up as though some work is copied (hubs $\mathrm{u}$ and $\mathrm{v}$ are doing likewise calculation by symmetry). These can be effortlessly determined by utilizing some need rules in light of hub ids.

\section{Performance Analysis}

In this segment, the execution of the proposed instrument is assessed utilizing system test system (ns2). In this test, the system incorporates 50 hubs sent arbitrarily in a 1000 meters $\times 1000$ meters field and the transmission reach is characterized 250 meters. There is no development of hubs and the foundation activity is produced haphazardly by an irregular generator gave by ns 2 . The CBR association with 4 parcels for every second are made and the extent of the bundle is 512 bytes. In the recreation, two wormhole hubs are made arbitrarily into the system and build up a passage between them utilizing embodiment.

Phase1: DATA TRANSMITTING

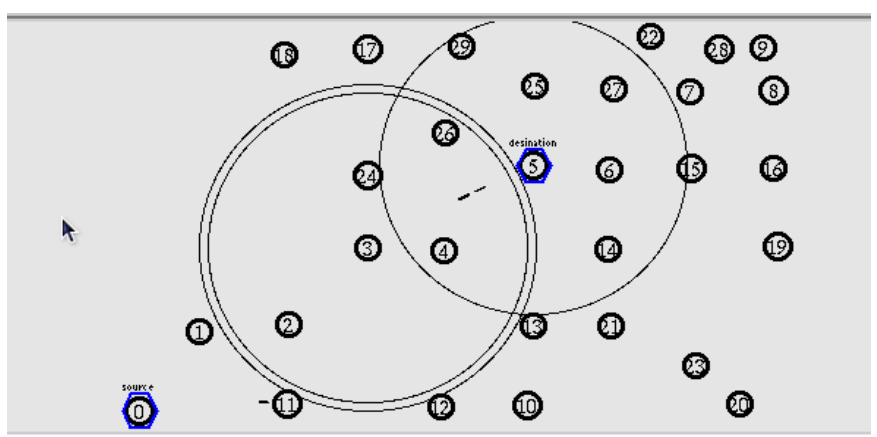

Figure 2. Data Transmission

Phase2: WORM HOLE ATTACK

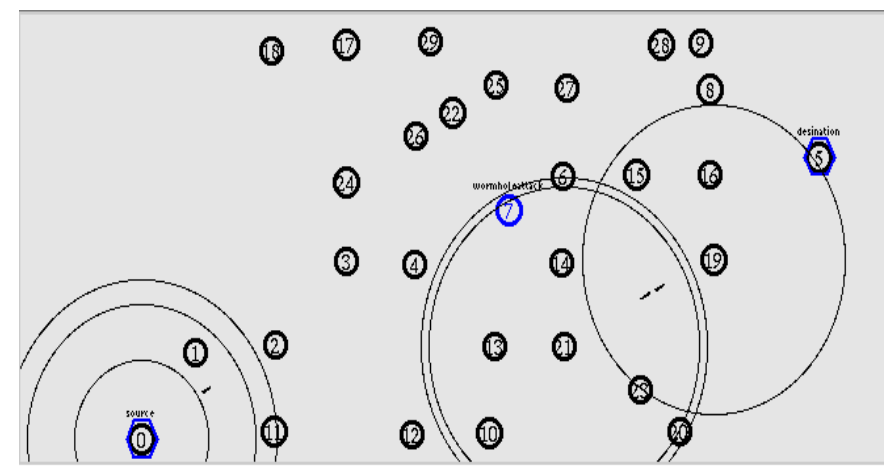

Figure 3. Wormhole Attack Identified

Phase3: WORMHOLE DETECTION 


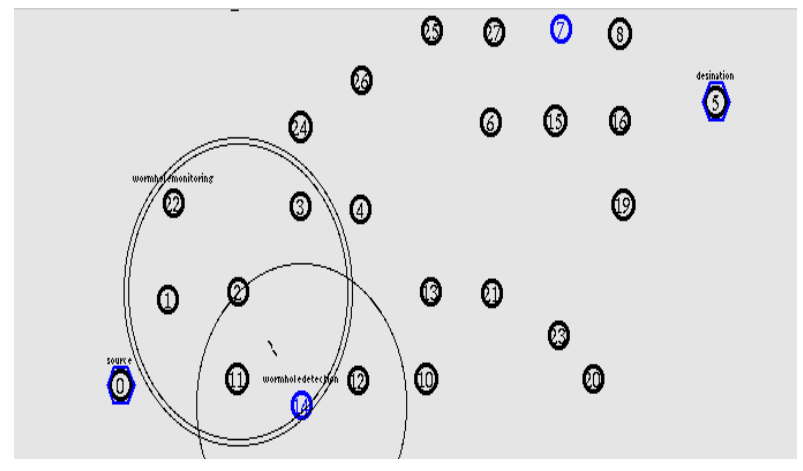

Figure 4. Wormhole Attack Detected and Recovered

WORMHOLE DETECTION:

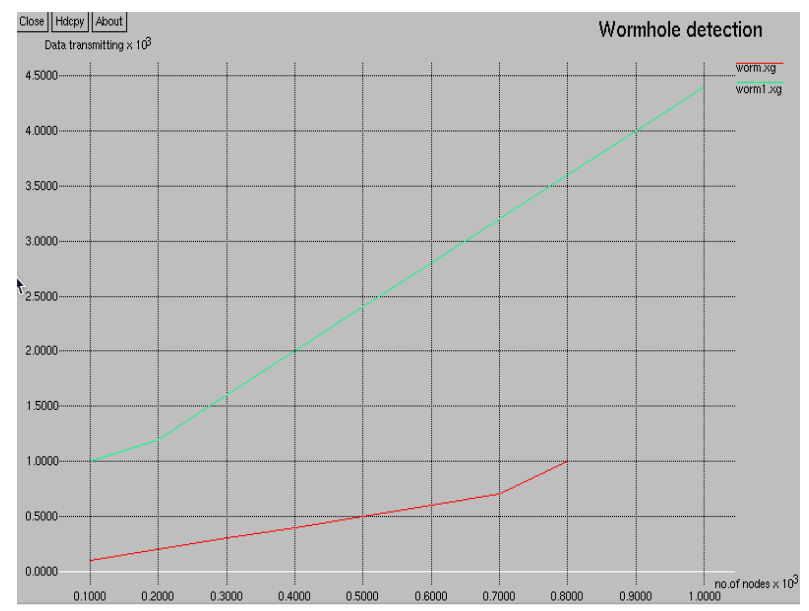

ENERGY CONSUMPTION:

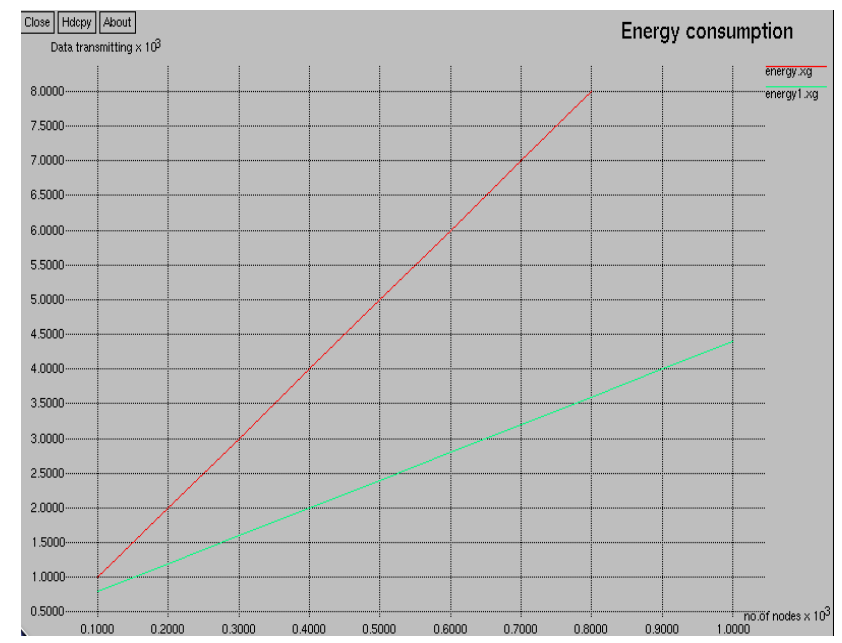




\section{PACKET LOSS:}

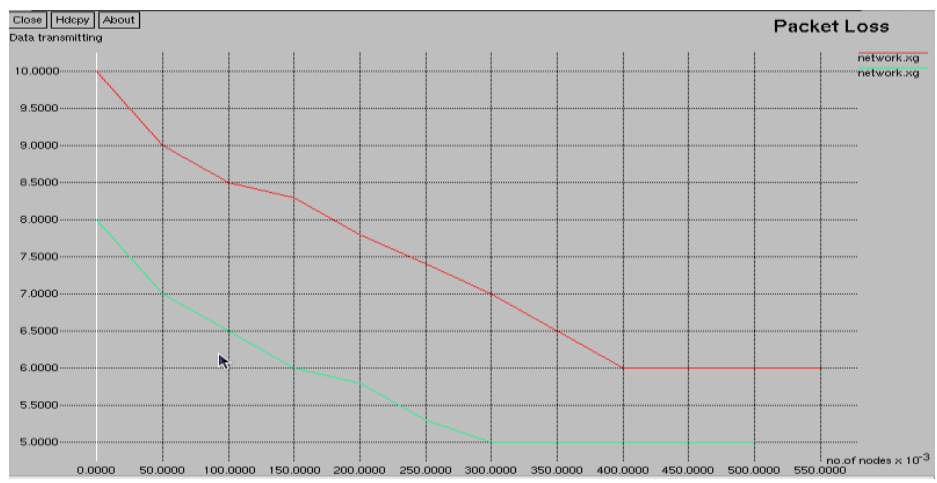

\section{Conclusion}

In this paper we propose a reasonable calculation for wormhole identification. The calculation is straightforward, restricted, and is general to hub conveyances and correspondence models. Our recreation results have affirmed a close impeccable discovery execution at whatever point the system is associated with a sufficiently high likelihood, for normal availability and hub dissemination models. We expect that this calculation will have a pragmatic use in true organizations to improve the power of remote systems against wormhole assaults.

\section{References}

[1] Y. C. Hu, A. Perrig and D. Johnson, "Packet leashes: a defense against wormhole attacks in wireless networks", In INFOCOM, (2003).

[2] L. Lazos, R. Poovendran, C. Meadows, P. Syverson and L.W. Chang, "Preventing Wormhole Attacks on Wireless Ad Hoc Networks: A Graph Theoretic Approach", In Proceedings of Wireless Communications and Networking Conference, IEEE, (2005) March, pp. 1193-1199.

[3] P. Papadimitratos and Z. J. Haas, "Secure routing for mobile ad hoc networks", In SCS Communication Networks and Distributed Systems Modeling and Simulation Conference (CNDS 2002), (2002).

[4] A. A. Pirzada and C. McDonald, "Circumventing Sinkholes and Wormholes in Wireless Sensor Networks", In International Work-shop on Wireless Ad Hoc networks, (2005) May, pp. 132-150.

[5] L. Qian, N. Song and X. Li, "Detecting and locating wormhole attacks in Wireless Ad Hoc Networks through statistical analysis of multi-path", In IEEE Wireless Communications and Networking Conference - WCNC, (2005).

[6] K. Sanzgiri, B. Dahill, B. Levine and E. Belding-Royer, "A secure routing protocol for ad hoc networks", In International Conference on Network Protocols (ICNP), (2002) November.

[7] W. Sharif and C. Leckie. "New Variants of Wormhole Attacks for Sensor Networks", In the proceeding of the Australian Telecommunication Networks and Applications Conference, Melbourne Australia, (2006) December, pp. 288-292. 